\title{
On Motion around the Collinear Equilibrium Points in the Relativistic R3BP with a Smaller Triaxial Primary
}

\author{
Nakone Bello ${ }^{1, a^{*}}$ and Jagadish Singh ${ }^{2, b}$ \\ ${ }^{1}$ Department of Mathematics, Faculty of Science, Usmanu Danfodiyo University, Sokoto, \\ Nigeria. \\ ${ }^{2}$ Department of Mathematics, Faculty of Science, Ahmadu Bello University, Zaria, Nigeria. \\ abnakone@yahoo.com, bjgds2004@yahoo.com
}

Keywords: Celestial Mechanics, Triaxiality, Relativity, R3BP

\begin{abstract}
This paper studies the motion of an infinitesimal mass near the collinear equilibrium points in the framework of relativistic restricted three-body problem (R3BP) when the smaller primary is a triaxial body. It is observed that the positions of the collinear points are affected by the relativistic and triaxiality factors. The collinear points are found to remain unstable. Numerical studies in this connection, with the Sun-Earth, Sun-Pluto and Earth-Moon systems have been carried out to show the relativistic and triaxiality effects.
\end{abstract}

\section{Introduction}

The restricted three-body problem (R3BP) possesses five stationary solutions called Lagrangian points, three of which denoted by $L_{1}, L_{2}$ and $L_{3}$ known as collinear equilibria lying on the line joining the primaries, the other two denoted by $L_{4}$ and $L_{5}$ are called equilateral equilibria and make equilateral triangles with primaries. In general, the collinear points are unstable while equilateral points are stable for the mass ratio $\mu \leq 0.038520 \ldots$. (Szebehely [1])

Khanna and Bhatnagar [2] have discussed the stationary solutions of the planar restricted three-body problem when the smaller primary is a triaxial rigid body and a bigger one as an oblate spheroid. They found that the collinear points are unstable, while the triangular points are stable for the mass parameter $0 \leq \mu<\mu_{c r i t}$ (the critical mass parameter). Sharma and SubbRao [3] investigated numerically the collinear libration points, by taking the oblateness of the primaries in consideration for 19 systems. They found that in some of the systems the shifts are significant. These equilibria are shown to be unstable in general, though the existence of conditional, infinitesimal (linearized) periodic orbits around them can be established, in the usual way. They also showed that the eccentricity and synodic period of these orbits are functions of oblateness. Numerical study, in this connection, with the above systems, revealed that the orbits around the libration point which is farthest from the primary whose oblateness effect is included, exhibit a different trend from those around the other two points. Abdul Raheem and Singh [4] have investigated the stability of equilibrium points under the influence of small perturbations in Coriolis and Centrifugal forces, together with the effects of oblateness and radiation pressures of the primaries. They have found that the collinear points remain unstable while the triangular points are stable for $0 \leq \mu<\mu_{c}$ and unstable for $\mu_{c} \leq \mu \leq \frac{1}{2}$, where $\mu_{c}$ is the critical mass parameter depending upon the Coriolis force, centrifugal force, oblateness and radiation pressure of the primaries.

Singh and Umar [5] investigated the motion of a test particle in the vicinity of a binary made of a triaxial primary and spherical companion moving along elliptic orbits about their common barycenter in the neighborhood of the collinear libration points. They obtained the analytical results and applied it to binary neutron stars consisting of a bigger triaxial primary and a spherical companion. 
Abouelmagd and El-Shaboury [6] studied the existence of libration points and their linear stability when three participating bodies are axisymmetric and the primaries are radiating, they found that the collinear points remain unstable, and the triangular points are stable for region $0 \leq \mu<\mu_{c}$. They also studied periodic orbits around the triangular points and found that these orbits are elliptical; the frequencies of long and short orbits of the periodic motion are affected by terms which involve the parameters that characterize the oblateness and radiation repulsive forces; they deduced that the period of long periodic orbits adjusts with the change in its frequency while the period of short periodic orbits will decrease.

The relativistic restricted three-body problem was basically originated by Brumberg [7] when he was studying the motion of Moon. After then a series of studies on the relativistic restricted three- body problem has been carried out.

Bhatnagar and Hallan [8] have studied the stability of triangular points of the same model problem and found that the triangular points are stable in the whole region $0 \leq \mu \leq \frac{1}{2}$ contrary to the classical case where they are stable for $\mu<\mu_{0}$ where $\mu_{0}=0.038521 \ldots$ is the Routh's critical mass value.

Ragos et al. [9] studied the existence, position and stability of collinear points of the relativistic R3BP.

Abd El- Bar and Abd El-Salam [10] investigated the relativistic effects on the equilibrium points. They obtained approximate locations of collinear and triangular points.

In recent years, the study of relativistic restricted three- body problem with perturbing forces such as radiation, oblateness, triaxiality, perturbations in the Coriolis and centrifugal forces has attracted many researchers.

Abd El-Bar and Abd El-Salam [11] computed the locations of collinear points in the photogravitational relativistic R3BP. Series forms of these locations are obtained as new results.

Katour et al. [12] studied the locations of triangular points in the relativistic restricted threebody problem with oblateness and photogravitational corrections.

Bello and Singh [13] studied the stability of triangular points in the relativistic R3BP with oblate primaries and bigger radiating.

Singh and Bello [14] studied the stability of $L_{4,5}$ in the photogravitational relativistic R3BP.

From authors' knowledge no work has been done on the stability of collinear points with perturbing force such as triaxiality in the relativistic R3BP. Hence it raised a curiosity in our minds to study the effect of triaxiality of the smaller primary on the locations and stability of collinear equilibrium points in the relativistic R3BP.

This paper is organized as follows: In Sect. 2, the equations governing the motion are presented; Sect. 3 describes the positions of collinear points, while their linear stability is analyzed in Sect.4. A numerical application are given in Sect. 5 and 6, respectively. Finally Sect. 7 conveys the main findings of this paper.

\section{Equations of Motion}

The pertinent equations of motion of the infinitesimal mass in the relativistic R3BP in a barycentric synodic coordinate $\operatorname{system}(\xi, \eta)$ and dimensionless variables is given by Brumberg [7] and Bhatnagar and Hallan [8] as: 


$$
\begin{aligned}
& \ddot{\xi}-2 n \dot{\eta}=\frac{\partial W}{\partial \xi}-\frac{d}{d t}\left(\frac{\partial W}{\partial \dot{\xi}}\right) \\
& \ddot{\eta}+2 n \dot{\xi}=\frac{\partial W}{\partial \eta}-\frac{d}{d t}\left(\frac{\partial W}{\partial \dot{\eta}}\right)
\end{aligned}
$$

with

$$
\begin{gathered}
W=\frac{1}{2}\left(\xi^{2}+\eta^{2}\right)+\frac{1-\mu}{\rho_{1}}+\frac{\mu}{\rho_{2}}+\frac{1}{c^{2}}\left[-\frac{3}{2}\left(1-\frac{1}{3} \mu(1-\mu)\right)\left(\xi^{2}+\eta^{2}\right)+\frac{1}{8}\left\{\dot{\xi}^{2}+\dot{\eta}^{2}+2(\xi \dot{\eta}-\eta \dot{\xi})+\left(\xi^{2}+\eta^{2}\right)\right\}^{2}\right. \\
+\frac{3}{2}\left(\frac{1-\mu}{\rho_{1}}+\frac{\mu}{\rho_{2}}\right)\left(\dot{\xi}^{2}+\dot{\eta}^{2}+2(\xi \dot{\eta}-\eta \dot{\xi})+\left(\xi^{2}+\eta^{2}\right)\right)-\frac{1}{2}\left(\frac{(1-\mu)^{2}}{\rho_{1}^{2}}+\frac{\mu^{2}}{\rho_{2}^{2}}\right)+\mu(1-\mu)\left\{\left(4 \dot{\eta}+\frac{7}{2} \xi\right)\left(\frac{1}{\rho_{1}}-\frac{1}{\rho_{2}}\right)\right. \\
\left.\left.-\frac{\eta^{2}}{2}\left(\frac{\mu}{\rho_{1}^{3}}+\frac{1-\mu}{\rho_{2}^{3}}\right)+\left(\frac{-1}{\rho_{1} \rho_{2}}+\frac{3 \mu-2}{2 \rho_{1}}+\frac{1-3 \mu}{2 \rho_{2}}\right)\right\}\right] \\
n=1-\frac{3}{2 c^{2}}\left(1-\frac{1}{3} \mu(1-\mu)\right) \\
\rho_{1}^{2}=(\xi+\mu)^{2}+\eta^{2} \\
\rho_{2}^{2}=(\xi+\mu-1)^{2}+\eta^{2}
\end{gathered}
$$

where $0<\mu \leq \frac{1}{2}$ is the ratio of the mass of the smaller primary to the total mass of the primaries; $\rho_{1}$ and $\rho_{2}$ are distances of the infinitesimal mass from the bigger and smaller primary, respectively; $n$ is the mean motion of the primaries; $c$ is the velocity of light.

We now introduce the triaxiality factors of the smaller primary with the help of the parameters $\sigma_{i}<<1, i=1,2$, where $\sigma_{1}=\frac{a^{2}-c^{2}}{5 R^{2}}, \sigma_{2}=\frac{b^{2}-c^{2}}{5 R^{2}}$ (McCuskey, [15]) with a, b, c as lengths of its semi-axes and $R$ is the dimensional distance between the primaries.

Ignoring second and higher powers of $\sigma_{i}$ and neglecting also their product, we take equations of motion as:

$$
\begin{gathered}
\ddot{\xi}-2 n \dot{\eta}=\frac{\partial W}{\partial \xi}-\frac{d}{d t}\left(\frac{\partial W}{\partial \dot{\xi}}\right) \\
\ddot{\eta}+2 n \dot{\xi}=\frac{\partial W}{\partial \eta}-\frac{d}{d t}\left(\frac{\partial W}{\partial \dot{\eta}}\right)
\end{gathered}
$$

where $W$ is the potential like function of the relativistic R3BP. As Katour et al. [12], we do not include the parameters $\sigma_{i}(i=1,2)$, in the relativistic part of $W$ since the magnitude of these terms is so small due to $c^{-2}$ where $c$ is the speed of light.

Hence, 


$$
\begin{aligned}
& W=\frac{1}{2}\left(1+\frac{3}{2}\left(2 \sigma_{1}-\sigma_{2}\right)\right)\left(\xi^{2}+\eta^{2}\right)+\frac{1-\mu}{\rho_{1}}+\frac{\mu}{\rho_{2}}+\frac{\mu}{2 \rho_{2}^{3}}\left(2 \sigma_{1}-\sigma_{2}\right)+\frac{3 \mu \eta^{2}}{2 \rho_{2}^{5}}\left(\sigma_{2}-\sigma_{1}\right) \\
& +\frac{1}{c^{2}}\left[-\frac{3}{2}\left\{1-\frac{1}{3} \mu(1-\mu)\right\}\left(\xi^{2}+\eta^{2}\right)+\frac{1}{8}\left\{\dot{\xi}^{2}+\dot{\eta}^{2}+2(\xi \dot{\eta}-\eta \dot{\xi})+\left(\xi^{2}+\eta^{2}\right)\right\}^{2}\right. \\
& +\frac{3}{2}\left(\frac{1-\mu}{\rho_{1}}+\frac{\mu}{\rho_{2}}\right)\left\{\dot{\xi}^{2}+\dot{\eta}^{2}+2(\xi \dot{\eta}-\eta \dot{\xi})+\left(\xi^{2}+\eta^{2}\right)\right\}-\frac{1}{2}\left(\frac{(1-\mu)^{2}}{\rho_{1}^{2}}+\frac{\mu^{2}}{\rho_{2}^{2}}\right) \\
& \left.+\mu(1-\mu)\left\{\left(4 \dot{\eta}+\frac{7}{2} \xi\right)\left(\frac{1}{\rho_{1}}-\frac{1}{\rho_{2}}\right)-\frac{\eta^{2}}{2}\left(\frac{\mu}{\rho_{1}^{3}}+\frac{1-\mu}{\rho_{2}^{3}}\right)+\left(\frac{-1}{\rho_{1} \rho_{2}}+\frac{3 \mu-2}{2 \rho_{1}}+\frac{1-3 \mu}{2 \rho_{2}}\right)\right\}\right],
\end{aligned}
$$

and $n$ is the perturbed mean motion of the primaries and is given by

$$
n=1+\frac{3}{4}\left(2 \sigma_{1}-\sigma_{2}\right)-\frac{3}{2 c^{2}}\left(1-\frac{1}{3} \mu(1-\mu)\right) .
$$

\section{Location of Collinear Points}

Equilibrium points are those points at which the centrifugal and gravitational forces balance out. That is no resultant force acts on the third infinitesimal body if placed there. Therefore, if it is placed at any of these points with zero velocity, it will stay there. In fact all derivatives of the coordinates with respect to the time are zero at these points. Therefore, the equilibrium points are solutions of equations

$$
W_{\xi}=0 \text { and } W_{\eta}=0 \text {. }
$$

$W_{\xi}$ and $W_{\eta}$ may be written as

$$
\begin{aligned}
& W_{\xi}=\xi-\frac{(1-\mu)(\xi+\mu)}{\rho_{1}^{3}}-\frac{\mu(\xi-1+\mu)}{\rho_{2}^{3}}+\left(3 \sigma_{1}-\frac{3}{2} \sigma_{2}\right) \xi-\frac{3 \mu(\xi-1+\mu)\left(2 \sigma_{1}-\sigma_{2}\right)}{2 \rho_{2}^{5}}-\frac{15 \mu(\xi-1+\mu)\left(\sigma_{2}-\sigma_{1}\right) \eta^{2}}{2 \rho_{2}^{7}} \\
& +\frac{1}{c^{2}}\left[-3 \xi\left\{1-\frac{\mu(1-\mu)}{3}\right\}+\frac{1}{2} \xi\left(\xi^{2}+\eta^{2}\right)-\frac{3}{2}\left(\xi^{2}+\eta^{2}\right)\left\{\frac{(1-\mu)(\xi+\mu)}{\rho_{1}^{3}}+\frac{\mu(\xi-1+\mu)}{\rho_{2}^{3}}\right\}+3\left(\frac{1-\mu}{\rho_{1}}+\frac{\mu}{\rho_{2}}\right) \xi\right. \\
& +\frac{(1-\mu)^{2}(\xi+\mu)}{\rho_{1}^{4}}+\frac{\mu^{2}(\xi-1+\mu)}{\rho_{2}^{4}}+\mu(1-\mu)\left\{\frac{7}{2}\left(\frac{1}{\rho_{1}}-\frac{1}{\rho_{2}}\right)+\frac{7}{2} \xi\left(-\frac{(\xi+\mu)}{\rho_{1}^{3}}+\frac{(\xi-1+\mu)}{\rho_{2}^{3}}\right)\right. \\
& \left.\left.+\frac{3}{2} \eta^{2}\left(\frac{\mu(\xi+\mu)}{\rho_{1}^{5}}+\frac{(1-\mu)(\xi-1+\mu)}{\rho_{2}^{5}}\right)+\frac{(\xi+\mu)}{\rho_{1}^{3} \rho_{2}}+\frac{(\xi-1+\mu)}{\rho_{1} \rho_{2}^{3}}-\frac{(3 \mu-2)(\xi+\mu)}{2 \rho_{1}^{3}}-\frac{(1-3 \mu)(\xi-1+\mu)}{2 \rho_{2}^{3}}\right\}\right]
\end{aligned}
$$

and

$$
W_{\eta}=\eta F,
$$

with

$$
\begin{aligned}
& F=\left(1-\frac{1-\mu}{\rho_{1}^{3}}-\frac{\mu}{\rho_{2}^{3}}\right)+\left(3 \sigma_{1}-\frac{3}{2} \sigma_{2}\right)+\frac{3 \mu}{\rho_{2}^{5}}\left(\frac{3}{2} \sigma_{2}-2 \sigma_{1}\right)-\frac{15 \mu\left(\sigma_{2}-\sigma_{1}\right) \eta^{2}}{2 \rho_{2}^{7}} \\
& \frac{1}{c^{2}}\left[-3\left(1-\frac{\mu(1-\mu)}{3}\right)+\frac{1}{2}\left(\xi^{2}+\eta^{2}\right)+3\left(\frac{1-\mu}{\rho_{1}^{3}}+\frac{\mu}{\rho_{2}^{3}}\right)-\frac{3}{2}\left(\xi^{2}+\eta^{2}\right)\left(\frac{1-\mu}{\rho_{1}^{3}}+\frac{\mu}{\rho_{2}^{3}}\right)+\left(\frac{(1-\mu)^{2}}{\rho_{1}^{4}}+\frac{\mu^{2}}{\rho_{2}^{4}}\right)\right. \\
& \left.+\mu(1-\mu)\left\{\frac{7}{2} \xi\left(-\frac{1}{\rho_{1}^{3}}+\frac{1}{\rho_{2}^{3}}\right)-\left(\frac{\mu}{\rho_{1}^{3}}+\frac{1-\mu}{\rho_{2}^{3}}\right)+\frac{3}{2} \eta^{2}\left(\frac{\mu}{\rho_{1}^{5}}+\frac{1-\mu}{\rho_{2}^{5}}\right)+\frac{1}{\rho_{1}^{3} \rho_{2}}+\frac{1}{\rho_{1} \rho_{2}^{3}}-\frac{(3 \mu-2)}{2 \rho_{1}^{3}}-\frac{(1-3 \mu)}{2 \rho_{2}^{3}}\right\}\right] .
\end{aligned}
$$


In order to find the collinear points, we put $\eta=0$ in equation (8). Their abscissae are the roots of the equation

$$
\begin{aligned}
& g(\xi)=\xi-\frac{(1-\mu)(\xi+\mu)}{\rho_{1}^{3}}-\frac{\mu(\xi-1+\mu)}{\rho_{2}^{3}}+\left(3 \sigma_{1}-\frac{3}{2} \sigma_{2}\right) \xi-\frac{3 \mu(\xi-1+\mu)\left(2 \sigma_{1}-\sigma_{2}\right)}{2 \rho_{2}^{5}} \\
& +\frac{1}{c^{2}}\left[-3 \xi\left(1-\frac{\mu(1-\mu)}{3}\right)+\frac{1}{2} \xi^{3}-\frac{3}{2} \xi^{2}\left\{\frac{(1-\mu)(\xi+\mu)}{\rho_{1}^{3}}+\frac{\mu(\xi-1+\mu)}{\rho_{2}^{3}}\right\}+3\left(\frac{1-\mu}{\rho_{1}}-\frac{\mu}{\rho_{2}}\right) \xi\right. \\
& +\frac{(1-\mu)^{2}(\xi+\mu)}{\rho_{1}^{4}}+\frac{\mu^{2}(\xi-1+\mu)}{\rho_{2}^{4}}+\mu(1-\mu)\left\{\frac{7}{2}\left(\frac{1}{\rho_{1}}-\frac{1}{\rho_{2}}\right)+\frac{7}{2} \xi\left(-\frac{(\xi+\mu)}{\rho_{1}^{3}}+\frac{(\xi-1+\mu)}{\rho_{2}^{3}}\right)\right. \\
& \left.\left.+\frac{\xi-1+\mu}{\rho_{1} \rho_{2}^{3}}+\frac{\xi+\mu}{\rho_{1}^{3} \rho_{2}}-\frac{(3 \mu-2)(\xi+\mu)}{2 \rho_{1}^{3}}-\frac{(1-3 \mu)(\xi-1+\mu)}{2 \rho_{2}^{3}}\right\}\right]=0
\end{aligned}
$$

with $\rho_{1}=|\xi+\mu|, \rho_{2}=|\xi-1+\mu|$.

To set the positions of collinear points on the $\xi$-axis, we divide the orbital plane into three parts: $\xi<\xi_{1}, \xi_{1}<\xi<\xi_{2}$ and $\xi_{2}<\xi$ with respect to the primaries, where $\xi_{1}=-\mu$ and $\xi_{2}=1-\mu$ as shown in Fig. 1 below.

$$
m_{1}=1-\mu \quad m_{2}=\mu \quad \lambda_{1} \quad L_{1}
$$

(a)

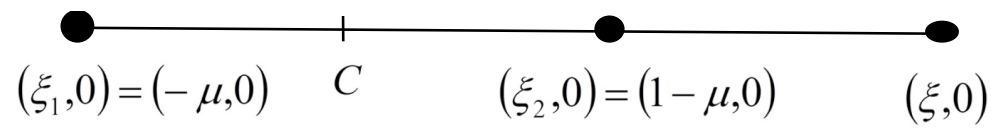

$$
m_{1}=1-\mu \quad L_{2} \quad \lambda_{2} \quad m_{2}=\mu
$$

(b)

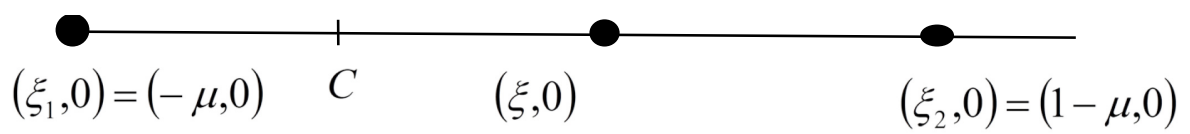

$$
L_{3} \quad 1-\lambda_{3} \quad m_{1}=1-\mu \quad m_{2}=\mu
$$

(c)

$$
\left(\xi_{1}, 0\right)=(-\mu, 0) \quad C \quad\left(\xi_{2}, 0\right)=(1-\mu, 0)
$$

Figure 1. Reference parameter for collinear Lagrangian points

Case 1. Position of $L_{1}\left(\xi>\xi_{2}\right)$ (see Fig. 1 (a))

Let $\xi-\xi_{2}=\lambda_{1} ; \xi-\xi_{1}=1+\lambda_{1} \Rightarrow \xi=1+\lambda_{1}+\xi_{1}$; since the distance between the primaries is unity, i.e. $\xi_{2}-\xi_{1}=1 \Rightarrow \xi_{1}=-\mu$ and $\xi_{2}=1-\mu$ then

$$
\xi=1+\lambda_{1}-\mu ; \rho_{1}=1+\lambda_{1} ; \rho_{2}=\lambda_{1} \text { with } \rho_{i}>0(i=1,2) .
$$

Now substituting equation (10) in (9), we obtain 


$$
\begin{aligned}
& \lambda_{1}^{10}+(6-3 \mu) \lambda_{1}{ }^{9}+\left(9+2 c^{2}-13 \mu-3 c^{2} \sigma_{2}+\mu^{2}+6 c^{2} \sigma_{1}\right) \lambda_{1}^{8}+ \\
& \left(24 c^{2} \sigma_{1}+2 \mu^{2}-12 c^{2} \sigma_{2}+3 \mu c^{2} \sigma_{2}-16 \mu+8 c^{2}-1+\mu^{3}-6 \mu c^{2} \sigma_{1}-2 \mu c^{2}\right) \lambda_{1}{ }^{7}+ \\
& \left(-18 c^{2} \sigma_{2}+9 \mu c^{2} \sigma_{2}+3 \mu^{3}-18 \mu c^{2} \sigma_{1}-6 \mu c^{2}+12 c^{2}-12+36 c^{2} \sigma_{1}\right) \lambda_{1}{ }^{6}+ \\
& \left(-7 \mu^{2}+24 c^{2} \sigma_{1}-18 \mu c^{2} \sigma_{1}+6 c^{2}+16 \mu-6 \mu c^{2}-9+3 \mu^{3}-12 c^{2} \sigma_{2}+9 \mu c^{2} \sigma_{2}\right) \lambda_{1}{ }^{5}+ \\
& \left(16 \mu-6 \mu c^{2} \sigma_{1}-14 \mu^{2}-3 c^{2} \sigma_{2}+6 c^{2} \sigma_{1}+3 \mu c^{2} \sigma_{2}-6 \mu c^{2}+3 \mu^{3}\right) \lambda_{1}^{4}+ \\
& \left(3 \mu c^{2} \sigma_{2}-12 \mu^{2}+15 \mu-6 \mu c^{2}+3 \mu^{3}-6 \mu c^{2} \sigma_{1}\right) \lambda_{1}^{3}+\left(5 \mu+9 \mu c^{2} \sigma_{2}-2 \mu c^{2}-18 \mu c^{2} \sigma_{1}+\mu^{3}\right) \lambda_{1}{ }^{2}+ \\
& \left(-18 \mu c^{2} \sigma_{1}+9 \mu c^{2} \sigma_{2}+2 \mu^{2}\right) \lambda_{1}+\left(3 \mu c^{2} \sigma_{2}-6 \mu c^{2} \sigma_{1}\right)=0 .
\end{aligned}
$$

In the presence of triaxiality effect only, we have

$$
\begin{aligned}
& \left(6 \sigma_{1}-3 \sigma_{2}+2\right) \lambda_{1}^{7}+\left(-6 \sigma_{1} \mu+18 \sigma_{1}-9 \sigma_{2}+3 \sigma_{2} \mu-2 \mu+6\right) \lambda_{1}^{6}+ \\
& \left(-12 \sigma_{1} \mu+18 \sigma_{1}-4 \mu-9 \sigma_{2}+6+6 \sigma_{2} \mu\right) \lambda_{1}^{5}+\left(-2 \mu+6 \sigma_{1}-6 \sigma_{1} \mu-3 \sigma_{2}+3 \sigma_{2} \mu\right) \lambda_{1}^{4} \\
& -4 \mu \lambda_{1}^{3}+\left(-2 \mu+3 \sigma_{2} \mu-6 \sigma_{1} \mu\right) \lambda_{1}^{2}+\left(-12 \sigma_{1} \mu+6 \sigma_{2} \mu\right) \lambda_{1}+3 \sigma_{2} \mu-6 \sigma_{1} \mu=0
\end{aligned}
$$

Case 2. Position of $L_{2}\left(\xi_{1}<\xi<\xi_{2}\right)$ (see Fig. 1 (b))

Let $\xi_{2}-\xi=\lambda_{2} ; \xi-\xi_{1}=1-\lambda_{2} \Rightarrow \xi=1-\lambda_{2}-\mu ; \rho_{1}=1-\lambda_{2} ; \quad \rho_{2}=\lambda_{2}$ with $\rho_{i}>0(i=1,2)$

Substituting equation (12) in (9), yields

$$
\begin{aligned}
& \lambda_{2}{ }^{10}+(-6+3 \mu) \lambda_{2}{ }^{9}+\left(\mu^{2}-13 \mu+2 c^{2}+9+6 c^{2} \sigma_{1}-3 c^{2} \sigma_{2}\right) \lambda_{2}{ }^{2}+ \\
& \left(-2 \mu^{2}-24 c^{2} \sigma_{1}+1+6 \mu c^{2} \sigma_{1}+2 \mu c^{2}+22 \mu-8 c^{2}-3 \mu c^{2} \sigma_{2}+12 c^{2} \sigma_{2}-\mu^{3}\right) \lambda_{2}{ }^{7}+ \\
& \left(3 \mu^{3}-18 \mu c^{2} \sigma_{1}+36 c^{2} \sigma_{1}+9 \mu c^{2} \sigma_{2}-6 \mu c^{2}-18 c^{2} \sigma_{2}+12 c^{2}-18 \mu-12\right) \lambda_{2}{ }^{6}+ \\
& \left(9+12 c^{2} \sigma_{2}+2 \mu c^{2}+18 \mu c^{2} \sigma_{1}+8 \mu-\mu^{2}-\mu^{3}-9 \mu c^{2} \sigma_{2}-24 c^{2} \sigma_{1}-6 c^{2}\right) \lambda_{2}{ }^{5}+ \\
& \left(6 \mu c^{2}-3 \mu^{3}-16 \mu-6 \mu c^{2} \sigma_{1}+18 \mu^{2}-3 c^{2} \sigma_{2}+6 c^{2} \sigma_{1}+3 \mu c^{2} \sigma_{2}\right) \lambda_{2}{ }^{4}+ \\
& \left(-6 \mu c^{2} \sigma_{1}-24 \mu^{2}+3 \mu c^{2} \sigma_{2}+15 \mu-6 \mu c^{2}+3 \mu^{3}\right) \lambda_{2}{ }^{3}+\left(-5 \mu+2 \mu c^{2}+18 \mu c^{2} \sigma_{1}-9 \mu c^{2} \sigma_{2}-\mu^{3}+12 \mu^{2}\right) \lambda_{2}{ }^{2} \\
& +\left(-18 \mu c^{2} \sigma_{1}+9 \mu c^{2} \sigma_{2}-2 \mu^{2}\right) \lambda_{2}+6 \mu c^{2} \sigma_{1}-3 \mu c^{2} \sigma_{2}=0
\end{aligned}
$$

In the presence of triaxiality effect only, we have

$$
\begin{aligned}
& \left(3 \sigma_{2}-6 \sigma_{1}-2\right) \lambda_{2}{ }^{7}+\left(18 \sigma_{1}-2 \mu-9 \sigma_{2}+3 \sigma_{2} \mu-6 \sigma_{1} \mu+6\right) \lambda_{2}{ }^{6}+\left(-18 \sigma_{1}+4 \mu+12 \sigma_{1} \mu+9 \sigma_{2}\right. \\
& \left.-6 \sigma_{2} \mu-6\right) \lambda_{2}{ }^{5}+\left(2 \mu+6 \sigma_{1}-6 \sigma_{1} \mu-3 \sigma_{2}+3 \sigma_{2} \mu\right) \lambda_{2}{ }^{4}-4 \mu \lambda_{2}{ }^{3}+\left(6 \sigma_{1} \mu-3 \sigma_{2} \mu+2 \mu\right) \lambda_{2}{ }^{2} \\
& +\left(6 \sigma_{2} \mu-12 \sigma_{1} \mu\right) \lambda_{2}+6 \sigma_{1} \mu-3 \sigma_{2} \mu=0
\end{aligned}
$$

Case 3. Position of $L_{3}\left(\xi<\xi_{1}\right)$ (see Fig. 1 (c))

Let the distance of the point $L_{3}$ from the bigger primary be $1-\lambda_{3}$.

Since $\xi_{2}-\xi_{1}=1 \Rightarrow \xi_{1}-\xi=1-\lambda_{3} ; \xi_{2}-\xi=2-\lambda_{3}$ and $\xi=\lambda_{3}-\mu-1 ; \rho_{1}=1-\lambda_{3} ; \rho_{2}=2-\lambda_{3}$ with $\rho_{i}>0(i=1,2)$

Substituting equation (14) in (9), we obtain 


$$
\begin{aligned}
& -\lambda_{3}^{10}+(3 \mu+14) \lambda_{3}{ }^{9}+\left(-\mu^{2}-6 c^{2} \sigma_{1}-41 \mu+3 c^{2} \sigma_{2}-81-2 c^{2}\right) \lambda_{3}^{8} \\
& +\left(2 \mu c^{2}+72 c^{2} \sigma_{1}+240 \mu+6 \mu c^{2} \sigma_{1}+247+14 \mu^{2}+24 c^{2}-3 \mu c^{2} \sigma_{2}-36 c^{2} \sigma_{2}-\mu^{3}\right) \lambda_{3}{ }^{7} \\
& +\left(11 \mu^{3}-404-22 \mu c^{2}-372 c^{2} \sigma_{1}-66 \mu c^{2} \sigma_{1}+186 c^{2} \sigma_{2}-84 \mu^{2}-124 c^{2}+33 \mu c^{2} \sigma_{2}-784 \mu\right) \lambda_{3}{ }^{6} \\
& +\left(255+306 \mu c^{2} \sigma_{1}+277 \mu^{2}-51 \mu^{3}+1080 c^{2} \sigma_{1}-153 \mu c^{2} \sigma_{2}-540 c^{2} \sigma_{2}+102 \mu c^{2}+358 c^{2}+1562 \mu\right) \lambda_{3}^{5} \\
& +\left(387 \mu c^{2} \sigma_{2}+131 \mu^{3}-1926 c^{2} \sigma_{1}-1950 \mu-262 \mu c^{2}+963 c^{2} \sigma_{2}+248-624 c^{2}-542 \mu^{2}-774 \mu c^{2} \sigma_{1}\right) \lambda_{3}{ }^{4} \\
& +\left(636 \mu^{2}-1080 c^{2} \sigma_{2}+2160 c^{2} \sigma_{1}-616-205 \mu^{3}+1507 \mu+410 \mu c^{2}+1146 \mu c^{2} \sigma_{1}+656 c^{2}-573 \mu c^{2} \sigma_{2}\right) \lambda_{3}^{3} \\
& +\left(480-673 \mu-398 \mu c^{2}-990 \mu c^{2} \sigma_{1}-432 \mu^{2}-384 c^{2}+744 c^{2} \sigma_{2}-1488 c^{2} \sigma_{1}+199 \mu^{3}+495 \mu c^{2} \sigma_{2}\right) \lambda_{3}{ }^{2} \\
& \left(158 \mu^{2}-231 \mu c^{2} \sigma_{2}+462 \mu c^{2} \sigma_{1}-288 c^{2} \sigma_{2}-144+224 \mu c^{2}+128 \mu-112 \mu^{3}+96 c^{2}+576 c^{2} \sigma_{1}\right) \lambda_{3} \\
& +\left(-56 \mu c^{2}-96 c^{2} \sigma_{1}+12 \mu-28 \mu^{2}+28 \mu^{3}-90 \mu c^{2} \sigma_{1}+48 c^{2} \sigma_{2}+45 \mu c^{2} \sigma_{2}\right)=0 .
\end{aligned}
$$

In the presence of triaxiality only, we have

$$
\begin{aligned}
& \left(6 \sigma_{1}-3 \sigma_{2}+2\right) \lambda_{3}^{7}+\left(-66 \sigma_{1}-2 \mu+33 \sigma_{2}-22-6 \sigma_{1} \mu+3 \sigma_{2} \mu\right) \lambda_{3}^{6}+\left(306 \sigma_{1}+20 \mu+60 \sigma_{1} \mu\right. \\
& \left.-153 \sigma_{2}+102-30 \sigma_{2} \mu\right) \lambda_{3}^{5}+\left(-82 \mu-256-246 \sigma_{1} \mu+387 \sigma_{2}-774 \sigma_{1}+123 \sigma_{2} \mu\right) \lambda_{3}^{4}+ \\
& \left(1152 \sigma_{1}+528 \sigma_{1} \mu+180 \mu-264 \sigma_{2} \mu-576 \sigma_{2}+368\right) \lambda_{3}^{3}+\left(309 \sigma_{2} \mu-288-618 \sigma_{1} \mu-1008 \sigma_{1}+\right. \\
& \left.504 \sigma_{2}-230 \mu\right) \lambda_{3}^{2}+\left(372 \sigma_{1} \mu+96-186 \sigma_{2} \mu-240 \sigma_{2}+168 \mu+480 \sigma_{1}\right) \lambda_{3}^{3}+\left(45 \sigma_{2} \mu-56 \mu+\right. \\
& \left.48 \sigma_{2}-96 \sigma_{1}-90 \sigma_{1} \mu\right)=0 .
\end{aligned}
$$

It should be noted that some of the above equations have more than one positive root but in each case there is only one physically acceptable root. It is also here pointed out that we have not considered the higher order relativistic corrections because $\xi_{i}, \frac{1}{c^{2}}<<1$.

\section{Stability of Collinear Points}

We investigate the stability of an equilibrium configuration, that is its ability to restrain the body motion in its vicinity. To do so we displace the infinitesimal body a little from an equilibrium point with small velocity. If its motion is rapid departure from vicinity of the point, we call such a position of equilibrium an unstable one. If the body oscillates about the point, it is said to be a stable position.

Following Singh and Bello [13], the characteristic equation is given by

$$
\left(P_{1} q_{2}-P_{2} q_{1}\right) \omega^{4}+\left(P_{1} q_{6}+P_{5} q_{2}+P_{3} q_{4}-P_{6} q_{1}-P_{2} q_{5}-P_{4} q_{3}\right) \omega^{2}+P_{5} q_{6}-P_{6} q_{5}=0,
$$

where

$$
\begin{aligned}
& P_{1}=1+W_{\dot{\xi} \xi}^{0}, P_{2}=W_{\dot{\eta} \xi}^{0}, P_{3}=W_{\xi \dot{\xi}}^{0}-W_{\xi \xi}^{0}=0, P_{4}=W_{\eta \dot{\xi}}^{0}-2 n-W_{\xi \dot{\eta}}^{0}, \\
& P_{5}=-W_{\xi \xi}^{0}, P_{6}=-W_{\xi \eta}^{0}, q_{1}=W_{\xi \dot{\xi}}^{0}, q_{2}=1+W_{\dot{\eta} \eta}^{0}, q_{3}=2 n+W_{\xi \dot{\eta}}^{0}-W_{\eta \dot{\xi}}^{0}, \\
& q_{4}=W_{\eta \dot{\eta}}^{0}-W_{\eta \dot{\eta}}^{0}=0, q_{5}=-W_{\xi \eta}^{0}, q_{6}=-W_{\eta \eta}^{0} .
\end{aligned}
$$

The second order partial derivative of $W$ are denoted by subscripts. The superscripts 0 indicates that the derivative is to be evaluated at the collinear equilibrium points $\left(\xi_{0}, \eta_{0}\right)$ under consideration. 
Studying the stability of the collinear points requires the study of motion in the proximity of these points, hence in this case the second order derivatives evaluated at $\left(\xi_{0}, \eta_{0}\right)$ are

$$
\begin{aligned}
& W_{\xi \xi}^{0}=1+\frac{3(1-\mu)(\xi+\mu)^{2}}{\rho_{1}^{5}}-\frac{(1-\mu)}{\rho_{1}^{3}}+\frac{3 \mu(\xi-1+\mu)^{2}}{\rho_{2}^{5}}-\frac{\mu}{\rho_{2}^{3}}+\left(3 \sigma_{1}-\frac{3}{2} \sigma_{2}\right)+\frac{15 \mu\left(2 \sigma_{1}-\sigma_{2}\right)(\xi-1+\mu)^{2}}{2 \rho_{2}^{7}} \\
& -\frac{3 \mu\left(2 \sigma_{1}-\sigma_{2}\right)}{2 \rho_{2}^{5}}+\frac{1}{c^{2}}\left[(\mu(1-\mu)-3)+\frac{3}{2} \xi^{2}+\left(\frac{9(1-\mu)(\xi+\mu)^{2}}{2 \rho_{1}^{5}}-\frac{3(1-\mu)}{2 \rho_{1}^{3}}-\frac{3 \mu}{2 \rho_{2}^{3}}+\frac{9 \mu(\xi-1+\mu)^{2}}{2 \rho_{2}^{5}}\right) \xi^{2}\right. \\
& +2\left(\frac{-3(1-\mu)(\xi+\mu)}{\rho_{1}^{3}}-\frac{3 \mu(\xi-1+\mu)}{\rho_{2}^{3}}\right) \xi+\frac{3(1-\mu)}{\rho_{1}}+\frac{3 \mu}{\rho_{2}}-\frac{4(1-\mu)^{2}(\xi+\mu)^{2}}{\rho_{1}^{6}}+\frac{(1-\mu)^{2}}{\rho_{1}^{4}}-\frac{4 \mu^{2}(\xi-1+\mu)^{2}}{\rho_{2}^{6}} \\
& +\frac{\mu^{2}}{\rho_{2}^{4}}+\mu(1-\mu)\left\{-\frac{7(\xi+\mu)}{\rho_{1}^{3}}+\frac{7(\xi-1+\mu)}{\rho_{2}^{3}}+\frac{7}{2}\left(\frac{3(\xi+\mu)^{2}}{\rho_{1}^{5}}-\frac{1}{\rho_{1}^{3}}+\frac{1}{\rho_{2}^{3}}-\frac{3(\xi-1+\mu)^{2}}{\rho_{2}^{5}}\right) \xi-\frac{3(\xi+\mu)^{2}}{\rho_{1}^{5} \rho_{2}}\right. \\
& -\frac{2(\xi+\mu)(\xi-1+\mu)}{\rho_{1}^{3} \rho_{2}^{3}}+\frac{1}{\rho_{1}^{3} \rho_{2}}-\frac{3(\xi-1+\mu)^{2}}{\rho_{1} \rho_{2}^{5}}+\frac{1}{\rho_{1} \rho_{2}^{3}}+\frac{3(3 \mu-2)(\xi+\mu)^{2}}{2 \rho_{1}^{5}}-\frac{(3 \mu-2)}{2 \rho_{1}^{3}}+\frac{3(1-3 \mu)(\xi-1+\mu)^{2}}{2 \rho_{2}^{5}} \\
& \left.\left.-\frac{(1-3 \mu)}{2 \rho_{2}^{3}}\right\}\right] \\
& W_{\eta \eta}^{0}=1-\frac{(1-\mu)}{\rho_{1}^{3}}-\frac{\mu}{\rho_{2}^{3}}+\left(3 \sigma_{1}-\frac{3}{2} \sigma_{2}\right)+\frac{3 \mu\left(3 \sigma_{2}-4 \sigma_{1}\right)}{2 \rho_{2}^{5}}+\frac{1}{c^{2}}\left[\mu(1-\mu)-3+\frac{1}{2} \xi^{2}+\left(-\frac{3(1-\mu)}{2 \rho_{1}^{3}}-\frac{3 \mu}{2 \rho_{2}^{3}}\right) \xi^{2}\right. \\
& +\frac{3(1-\mu)}{\rho_{1}}+\frac{3 \mu}{\rho_{2}}+\frac{(1-\mu)^{2}}{\rho_{1}^{4}}+\frac{\mu^{2}}{\rho_{2}^{4}}+\mu(1-\mu)\left\{\frac{7}{2}\left(-\frac{1}{\rho_{1}^{3}}+\frac{1}{\rho_{2}^{3}}\right) \xi-\frac{\mu}{\rho_{1}^{3}}-\frac{(1-\mu)}{\rho_{2}^{3}}+\frac{1}{\rho_{1}^{3} \rho_{2}}+\frac{1}{\rho_{1} \rho_{2}^{3}}\right. \\
& \left.\left.-\frac{(3 \mu-2)}{2 \rho_{1}^{3}}-\frac{(1-3 \mu)}{2 \rho_{2}^{3}}\right\}\right] \\
& W_{\xi \eta}^{0}=0 \\
& W_{\dot{\xi} \xi}^{0}=\frac{1}{c^{2}}\left[\frac{1}{2} \xi^{2}+\frac{3(1-\mu)}{\rho_{1}}+\frac{3 \mu}{\rho_{2}}\right] \\
& W_{\dot{\eta} \dot{\eta}}^{0}=\frac{1}{c^{2}}\left[\frac{3}{2} \xi^{2}+\frac{3(1-\mu)}{\rho_{1}}+\frac{3 \mu}{\rho_{2}}\right] \\
& W_{\dot{\eta} \dot{\xi}}^{0}=0 \\
& W_{\xi \xi}^{0}=0 \\
& W_{\eta \dot{\eta}}^{0}=0
\end{aligned}
$$

Next we have to prove that the discriminant $\Delta$ of (16) is positive at the collinear points $L_{i}(i=1,2,3)$

To show $\Delta$ is positive it is sufficient to show that

$$
T=-4\left(p_{1} q_{2}-p_{2} q_{1}\right)\left(p_{5} q_{6}-p_{6} q_{5}\right)>0 .
$$

$T$ can also be written as

$$
T=-4 W_{\xi \xi}^{0} W_{\eta \eta}^{0}\left(1+W_{\dot{\xi} \xi}^{0}\right)\left(1+W_{\dot{\eta} \dot{\eta}}^{0}\right)
$$

From (20) and (21) it clear that $\left(1+W_{\dot{\xi} \xi}^{0}\right)>0$ and $\left(1+W_{\dot{\eta} \dot{\eta}}^{0}\right)>0$.

Now we have to study the signs of $W_{\xi \xi}^{0}$ and $W_{\eta \eta}^{0}$ at the collinear points $L_{i}(i=1,2,3)$. 
Firstly we will do this at $L_{1}$, since the coordinate of this point is $\left(1+\lambda_{1}-\mu, 0\right)$, then $\rho_{1}=1+\lambda_{1}$ and $\rho_{2}=\lambda_{1}$ where $0<\lambda_{1}<<1$, hence we can write $W_{\xi \xi}^{0}$ and $W_{\eta \eta}^{0}$ as a function in $\lambda_{1}$ say $h\left(\lambda_{1}\right)$ and $f\left(\lambda_{1}\right)$, respectively. Therefore, in this case from $(17), h\left(\lambda_{1}\right) \cong h\left(0^{+}\right)=+\infty$ and from (18), $f\left(\lambda_{1}\right) \cong f\left(0^{+}\right)=-\infty$, hence $W_{\xi \xi}^{0}>0$ and $W_{\eta \eta}^{0}<0$ in which $W_{\xi \xi}^{0} W_{\eta \eta}^{0}<0$ and consequently $T>0$. Hence the discriminant of the equation (16) is positive, and the characteristic roots can be written as $\omega_{1,2}= \pm i \sigma, \omega_{3,4}= \pm i \tau$ where $\sigma$ and $\tau$ are real.

Thus $\omega_{1,2}$ are real and $\omega_{3,4}$ are pure imaginary, hence the motion around the collinear point $L_{1}$ is unbounded and the solution is unstable.

Similarly, it can be shown that the points $L_{2}, L_{3}$ are also unstable.

\section{Numerical Results}

We have used equations (11), (13), (15), (11a), (13a), (15a) respectively to compute the positions of the collinear points $L_{1}, L_{2}, L_{3}$ for the Sun-Earth system, Earth-Moon system and SunPluto system. We have taken five different cases of different set of semi-axes in $\mathrm{km}$. $(\mathrm{a}, \mathrm{b}, \mathrm{c})$ of the smaller primary. For the Sun-Earth system, we take (6400, 6400, 6400), (6400, 6390, 6380), (6400, $6380,6360),(6400,6370,6340)$ and $(6400,6360,6320)$, for the Earth -Moon system, we take $(1738,1738,1738),(1738,1728,1718)$,

$(1738,1718,1698),(1738,1708,1678),(1738,1698,1658)$ and for the Sun-Pluto system, we take $(3000,3000,3000),(3000,2990,2980)$,

$(3000,2980,2960),(3000,2970,2940),(3000,2960,2920)$. Some of the data has been borrowed from Ragos et al. [9] and Sharma and Subba Rao [3]

In Tables 1-3, we present these positions. The corresponding positions of the classical problem are also included. We also include the corresponding positions in the presence of triaxiality effect only (second entry in table for each system).

Table 1. Sun-Earth system, $\mu=0.000003003500, c=10064.84, R=149597870.61 \mathrm{~km}$

\begin{tabular}{|c|c|c|c|c|c|c|}
\hline parameter & Classical & case 1 & case 2 & case 3 & case 4 & case 5 \\
\hline$\sigma_{1}$ & & 0 & $2.284 \times 10^{-12}$ & $4.561 \times 10^{-12}$ & $6.831 \times 10^{-12}$ & $9.094 \times 10^{-12}$ \\
\hline$\sigma_{2}$ & & 0 & $1.141 \times 10^{-12}$ & $2.277 \times 10^{-12}$ & $3.48 \times 10^{-12}$ & $4.533 \times 10^{-12}$ \\
\hline$L_{1}$ & 1.01003413809074 & 1.01003413806000 & 1.01003413823000 & 1.01003413840000 & 1.01003413857000 & 1.01003413874000 \\
\hline$L_{2}$ & 0.99002657245077 & 0.99002657248300 & 1.01003413826000 & 1.01003413843000 & 1.01003413860000 & 1.01003413877000 \\
\hline$L_{3}$ & -1.00000125145833 & -1.00000125145831 & -1.000001251456060 & -1.000000125145489 & -1.000000125145319 & -1.000000125145148 \\
& & & -1.00000125145662 & -1.00000125145491 & -1.00000125145321 & -1.00000125145151 \\
\hline
\end{tabular}

Table 2. Sun-Pluto system, $\mu=0.00000006500, c=63280.18, R=5900 \times 10^{6} \mathrm{~km}$

\begin{tabular}{|c|c|c|l|c|c|c|}
\hline parameter & Classical & case 1 & case 2 & case 3 & case 4 & case 5 \\
\hline$\sigma_{1}$ & & 0 & $6.871588624 \times 10^{-16}$ & $1.369721344 \times 10^{-15}$ & $2.047687446 \times 10^{-15}$ & $2.721057167 \times 10^{-15}$ \\
\hline$\sigma_{2}$ & & 0 & $3.430048837 \times 10^{-16}$ & $6.825624820 \times 10^{-16}$ & $1.018672795 \times 10^{-15}$ & $1.351335823 \times 10^{-15}$ \\
\hline$L_{1}$ & 1.00129454074324 & 1.00129454074300 & 1.00129454074400 & 1.00129454074400 & 1.00129454074400 & 1.00129454074500 \\
\hline$L_{2}$ & 0.99870656252876 & 0.99870656252900 & 0.99870654074600 & 1.00129454074400 & 1.00129454074400 & 1.00129454074500 \\
\hline$L_{3}$ & -1.0000000270833 & -1.0000000270833 & -1.0000000270833 & -1.00000000270833 & -1.0000000270833 & -1.00000000270833 \\
\hline
\end{tabular}


Table 3. Earth-Moon system, $\mu=0.0121314293, c=292624.8185, R=384000 \mathrm{~km}$

\begin{tabular}{|c|c|c|c|c|c|c|}
\hline parameter & Classical & case 1 & case 2 & case 3 & case 4 \\
\hline$\sigma_{1}$ & & 0 & $9.375000000 \times 10^{-8}$ & $1.864149306 \times 10^{-7}$ & $2.779947917 \times 10^{-7}$ & $3.684895833 \times 10^{-7}$ \\
\hline$\sigma_{2}$ & & 0 & $4.673936632 \times 10^{-8}$ & $9.266493056 \times 10^{-8}$ & $1.377766927 \times 10^{-7}$ & $1.820746528 \times 10^{-7}$ \\
\hline$L_{1}$ & 1.155608442501 & 1.155608444240 & 1.155608847700 & 1.155609249000 & 1.155609646500 & 1.155610040000 \\
& & & 1.155608847700 & 1.155609249000 & 1.155609646400 & 1.155610040000 \\
\hline$L_{2}$ & 0.837009426190 & 0.837009426300 & 0.837008972600 & 0.837008523300 & 0.837008078400 & 0.837007637900 \\
\hline$L_{3}$ & -1.00505466491 & -1.005054664477 & -1.005054594295 & -1.005054524786 & -1.005054455958 & -1.005054387803 \\
& & & -1.005054594296 & -1.005054524791 & -1.005054455960 & -1.00505438780 \\
\hline
\end{tabular}

\section{Discussion}

Equations (5)-(6) describe the motion of a third body under the influence of the triaxiality of the smaller primary and relativistic effect. Equations (11), (13), (15) give respective position of the collinear equilibrium points $L_{1}, L_{2}, L_{3}$ which are affected by the relativistic and triaxiality factors. Equations (11a), (13a), (15a) give their positions in the presence of triaxiality only. It can be seen in section 4 that the relativistic and triaxiality factors are unable to change the instability character of the collinear points. For the Sun-Earth system in the absence of triaxiality $\left(\sigma_{1}=\sigma_{2}=0\right)$ the numerical results of the present study are in agreement with those of Ragos et al. [9]. This agreement can be easily seen in table 1 when the notations $L_{1}$ and $L_{2}$ are interchanged.

For the Sun-Earth system it can be observed for the Sun-Earth system from table 1 that $L_{1}$ moves towards the origin from the classical position due to relativistic effect only, whereas it moves from the classical position in the direction of the positive $\xi$-axis. The triaxiality has more shift than that of the joint effect.

$L_{2}$ moves along the positive $\xi$-axis from the classical position due to relativistic effect only, whereas it moves towards the origin from the classical case due to triaxiality alone or both. $L_{3}$ has a shift towards the origin due to triaxiality. It has also a shift towards the origin due to the joint effect. This shift is almost same as that of triaxiality. The relativistic shift in comparison with that of triaxiality is not remarkable.

For the Sun-Pluto system, it is observed from table 2 that the point $L_{1}$ has a very small shift towards the smaller primary from the classical position due to relativistic effect. The triaxiality shifts $L_{1}$ away from the classical position. This shift is almost similar as that of joint effect. The point $L_{2}$ has a very small shift in the direction of the positive $\xi$-axis from the classical position due to relativistic effect alone. The triaxiality shifts $L_{2}$ towards the origin from the classical position. The similar shift is also seen due to the joint effect. The classical position of $L_{3}$ does not change due to triaxiality or relativistic or both effects.

For the Earth -Moon system, it can be seen from Table 3 that $L_{1}$ moves towards the origin from the classical position due to relativistic effect. The triaxiality shifts it from the classical position away from the origin. The similar shift is also observed due to the joint effect. $L_{2}$ and $L_{3}$ all move towards the origin from the classical position due to relativistic or triaxiality or both effects.

\section{Conclusion}

By considering the smaller primary as triaxial rigid body, we have determined the positions of collinear points and have examined their linear stability. It is found that their positions are both affected by the relativistic terms and triaxiality parameters. This is confirmed from the tables. It is further observed that in spite of the introduction of relativistic and triaxiality coefficients, the 
collinear points remain unstable. Numerical investigations on this model by considering the SunEarth, Sun-Pluto and Earth-Moon systems have been performed to show the relativistic and triaxiality effects on collinear points

\section{References}

[1] V. Szebehely, Theory of orbits. The restricted problem of three- bodies, Academic Press, New York, 1967.

[2] M. Khanna, K.B. Bhatnagar, Existence and stability of libration points in the restricted threebody problem when the smaller primary is triaxial rigid body and the bigger one an oblate spheroid, Indian J. Pure Appl. Math. 7 (1999) 721-733.

[3] R.K. Sharma, P.V. SubbaRao, Collinear equilibria and their characteristic exponents in the restricted three-body problem when the primaries are oblate spheroids, Celest. Mech. 12 (1975) 189-201.

[4] A. AbdulRaheem, J. Singh, Combined effects of perturbations, radiation, and oblateness on the stability of equilibrium points in the restricted three-body problem, The Astronomical Journal. 131(3) (2006) 1880-1885.

[5] J. Singh, A. Umar, On motion around libration points in the elliptical R3BP with a bigger triaxial primary, New Astronomy. 29 (2014) 36-41.

[6] E.I. Abouelmagd, S.M. El-Shaboury, Periodic orbits under combined effects of oblateness and radiation in the restricted problem of three bodies, Astrophys. Space Sci. 341(2) (2012) 331-341.

[7] V.A. Brumberg, Relativistic celestial mechanics, Nauka, Moscow, USSR, 1972.

[8] K.B. Bhatnagar, P.P. Hallan, Existence and stability of $L_{4,5}$ in the relativistic restricted threebody problem, Celest. Mech. Dyn. Astron. 69(3) (1998) 271-281.

[9] O. Ragos et al., On the equilibrium points of the relativistic restricted three-body problem, Nonlinear Analysis. 47(5) (2001) 3413-3418.

[10] S.E. Abd El-Bar, F.A. Abd El-Salam, Computation of the locations of the libration points in the relativistic restricted three-body problem, American Journal of Applied Sciences. 9(5) (2012) 659-665.

[11] S.E. Abd El-Bar, F.A. Abd El-Salam, Analytical and semi analytical treatment of the collinear points in the photogravitational relativistic R3BP, Mathematical Problems in Engineering. 2013 (2013) Article ID 794734.

[12] D.A. Katour, F.A. Abd El-Salam, M.O. Shaker, Relativistic restricted three-body problem with oblateness and photo-gravitational corrections to triangular equilibrium points, Astrophys. Space Sci. 351(1) (2014) 143-149.

[13] N. Bello, J. Singh, On the stability of triangular points in the relativistic R3BP with oblate primaries and bigger radiating, Advances in Space Research. 57(2) (2016) 576-587.

[14] J. Singh, N. Bello, On the Stability of $L_{4,5}$ in the Photogravitational Relativistic R3BP, Differential Equations and Dynamical Systems. 25(1) (2017) 29-37.

[15] S.W. McCuskey, Introduction to celestial mechanics, Reading, Mass., Addison-Wesley Pub. Co., 1963. 